\title{
A rare presentation of pulmonary sarcoidosis as a solitary lung mass: a case report
}

\author{
Dylan W. Kelleher ${ }^{1}$, Madeleine Yaggi ${ }^{1}$, Robert Homer ${ }^{2}$, Erica L. Herzog ${ }^{1}$ and Changwan Ryu ${ }^{1 *}$
}

\begin{abstract}
Background: Sarcoidosis is a multisystem, chronic granulomatous disease of unknown etiology that predominantly affects the lungs. Pulmonary sarcoidosis classically presents with constitutional symptoms and computed tomographic scan findings of bilateral, symmetric micronodules in a peribronchovascular distribution with upper and middle lung zone predominance accompanied by bilateral, symmetric hilar lymphadenopathy. A solitary lung mass is a rare finding for pulmonary sarcoidosis, and with its associated constitutional symptoms, it strongly mimics a malignancy. We aimed to provide further insight into the broad differential diagnosis of a lung mass by describing our experiences in the care of a patient who presented with clinical and radiographic features of lung cancer who was ultimately found to have an atypical manifestation of stage II pulmonary sarcoidosis.
\end{abstract}

Case presentation: A 44-year-old African American woman with a history of childhood asthma and type 2 diabetes mellitus presented with shortness of breath. After being treated for a presumed asthma exacerbation with prednisone, she experienced worsening dyspnea, night sweats, and unintentional weight loss. Further evaluation revealed a large left lower lobe mass and hilar lymphadenopathy. A computed tomography-guided biopsy of the lung mass revealed a multifocal non-necrotizing granuloma with multinucleated giant cells. Although consistent with sarcoidosis, this finding could represent a sarcoid-like reaction secondary to an occult malignancy. A more extensive repeat biopsy via bronchoscopy and mediastinoscopy revealed granulomatous inflammation without evidence of malignancy or infection. These procedures confirmed the diagnosis of pulmonary sarcoidosis, and she was started on treatment with high-dose prednisone. Her treatment course was complicated by hyperglycemia necessitating insulin therapy, but after 3 months of therapy, she reported improvement in her dyspnea, and repeat imaging revealed a significant decrease in the size of the lung mass and lymphadenopathy. Given her clinical and radiographic response, she was continued on a prednisone taper.

Conclusions: Atypical manifestations of pulmonary sarcoidosis are diagnostically challenging because the clinical and radiographic features of the disease mimic those of a malignancy. We aimed to illustrate a unique etiology of a lung mass and the importance of maintaining a broad differential diagnosis. Nonetheless, with the possibility of a malignancy, a high index of suspicion is necessary for timely diagnosis and optimal management.

Keywords: Pulmonary sarcoidosis, Lung mass, Sarcoid-like reaction

\footnotetext{
* Correspondence: changwan.ryu@yale.edu

${ }^{1}$ Department of Internal Medicine, Section of Pulmonary, Critical Care and

Sleep Medicine, Yale University School of Medicine, 300 Cedar Street, TAC

441 South, P.O. Box 208057, New Haven, CT 06520, USA

Full list of author information is available at the end of the article
}

(c) The Author(s). 2018 Open Access This article is distributed under the terms of the Creative Commons Attribution 4.0 International License (http://creativecommons.org/licenses/by/4.0/), which permits unrestricted use, distribution, and reproduction in any medium, provided you give appropriate credit to the original author(s) and the source, provide a link to the Creative Commons license, and indicate if changes were made. The Creative Commons Public Domain Dedication waiver (http://creativecommons.org/publicdomain/zero/1.0/) applies to the data made available in this article, unless otherwise stated. 


\section{Background}

Sarcoidosis is a systemic, chronic granulomatous disease of unknown etiology that commonly affects the lungs [1]. Whereas some patients with pulmonary sarcoidosis are asymptomatic, many report cough, dyspnea, fatigue, unintentional weight loss, and night sweats [2]. Because of its nonspecific symptomology, computed tomographic (CT) scans are instrumental for diagnosis $[1,2]$. Common findings include bilateral, symmetric micronodules in a peribronchovascular distribution with upper and middle lung zone predominance accompanied by bilateral, symmetric hilar lymphadenopathy [3]. However, atypical radiographic patterns of pulmonary sarcoidosis [3] prove to be diagnostically challenging, particularly in the rare cases of mass lesions, because the associated constitutional symptoms strongly mimic a malignancy [4]. We aimed to provide further insight into the broad differential diagnosis of a lung mass by describing our experiences in the care of a patient who presented with clinical and radiographic features of lung cancer who was ultimately found to have a rare manifestation of stage II pulmonary sarcoidosis.

\section{Case presentation}

A 44-year-old African American woman with a past medical history significant for childhood asthma and type 2 diabetes mellitus presented to our institution with dyspnea. One month prior to presentation, she complained of shortness of breath that was presumed to be due to an asthma exacerbation and was treated with an empiric, 2 -week course of prednisone. She noted that her breathing improved while on prednisone, but she returned to our emergency department with worsening dyspnea once prednisone was tapered off. Her review of systems revealed night sweats for 2 months and an unintentional weight loss of 15 pounds.

Her vital signs and results of her physical examination were normal. Laboratory testing revealed normal complete blood count, chemistry panel, calcium, and vitamin D values. A chest radiograph revealed a masslike opacity in the left lower lobe, which was followed with a chest CT scan that revealed a large, $6.7 \times 5.4 \times 9.9$-cm left lower lobe mass (Fig. 1a) and hilar lymphadenopathy (Fig. 1b). Given the patient's ongoing constitutional symptoms, there was significant concern for a pulmonary malignancy; the mass was felt to be too distal for transbronchial biopsies via bronchoscopy, and a computed tomography-guided biopsy of the lung mass was performed, which revealed multifocal non-necrotizing granulomas with multinucleated giant cells without evidence of malignancy or active infection. Although the biopsy was consistent with sarcoidosis, this finding could represent a sarcoid-like reaction secondary to an occult malignancy. Subsequently, a repeat biopsy of the mass was successfully attempted via transbronchial biopsy via bronchoscopy, and a mediastinoscopy was performed to obtain sufficient tissue for a definitive diagnosis. The lung mass biopsy was again consistent with granulomatous inflammation without evidence of malignancy or active infection, and the lymph node biopsy (station 4R) revealed active granulomatous inflammation (Fig. 2).

Following these two procedures, we were confident in our diagnosis of pulmonary sarcoidosis. Given her debilitating symptoms, she was started on treatment with prednisone $60 \mathrm{mg}$ daily with appropriate prophylaxis. Within 1 month of treatment, she began to experience improvement of her dyspnea; however, her glucose control deteriorated to a point where she required insulin to manage her diabetes. A repeat $\mathrm{CT}$ scan after 3 months of high-dose prednisone revealed a significant decrease in the size of the lung mass, which measured $4.7 \times 3.1 \times 5.8 \mathrm{~cm}$ (Fig. 3a), and lymphadenopathy (Fig. 3b). Although we considered starting a steroid-sparing agent, we continued her on a prednisone taper, given her robust clinical and radiographic response.

\section{Discussion}

Atypical radiographic presentations of pulmonary sarcoidosis occur in $15-25 \%$ of patients [3]. These findings can include diffuse ground-glass opacities, honeycombing, multiple nodules, or necrotizing consolidations [3].

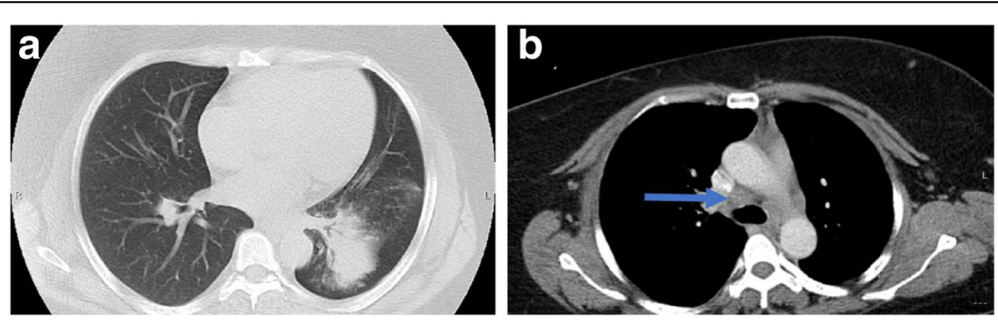

Fig. 1 Chest computed tomography reveals a large left lower lobe lung mass. On admission, the patient was found to have (a) a $6.7 \times 5.4 \times 9.9-\mathrm{cm}$ left lower lobe mass and (b) hilar lymphadenopathy (arrow) 


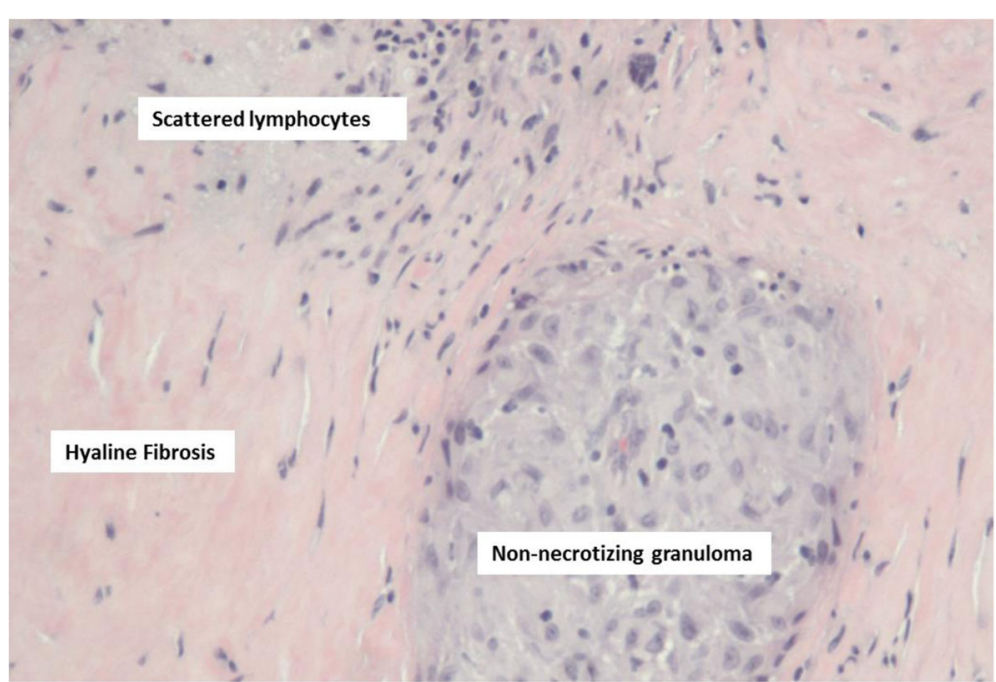

Fig. 2 Biopsy of hilar lymph node revealed granulomatous inflammation. High-power view of the right hilar lymph node (station 4R) biopsy shows non-necrotizing granuloma with scattered lymphocytes and surrounding fibrosis

A large, solitary lung mass, as described in our patient's case, has rarely been reported, and its incidence is unknown; it is likely to be the result of individual granulomas that have coalesced to produce the appearance of a mass [5].

Because solitary lung masses with mediastinal lymphadenopathy are the hallmarks of lung cancer, obtaining a definitive diagnosis in cases of atypical pulmonary sarcoidosis is diagnostically challenging. Prior case reports described the need for a surgical lung biopsy to obtain sufficient tissue for a definitive diagnosis $[4,6]$, which was the case in our patient. However, the presence of granulomas does not necessarily rule out a malignancy, because non-caseating granulomas have been reported in some instances of small cell lung cancer [7]. Moreover, the presence of non-caseating granulomas in association with a malignancy, described as a sarcoid-like reaction, has been shown to occur adjacent to the site of the malignancy or around local lymph nodes $[7,8]$. Sarcoid-like reactions, which are histologically indistinguishable from granulomas found in usual cases of sarcoidosis, are believed to be the result of an immunological response to local tumor products [8]. They occur in the context of both hematologic malignancies and solid tumors; one study demonstrated an association between sarcoid-like reactions and lung cancer [8]. In addition, sarcoid-like reactions occur in response to microbial infection, such as leishmaniasis, tuberculosis, and coccidioidomycosis [9]. Thus, in cases of atypical sarcoidosis, biopsies from two noncontiguous sites are recommended to rule out an active malignancy or infection [10].

Although prednisone is the first-line treatment for sarcoidosis [1, 2], in cases with significant adverse reactions, such as the one presented here, steroidsparing agents can be considered. Methotrexate is a commonly chosen second-line agent because it has been shown to be effective in decreasing the necessary dose of prednisone when used in combination [11]. For refractory cases, leflunomide and tumor necrosis factor- $\alpha$ antagonists, such as infliximab, adalimumab,

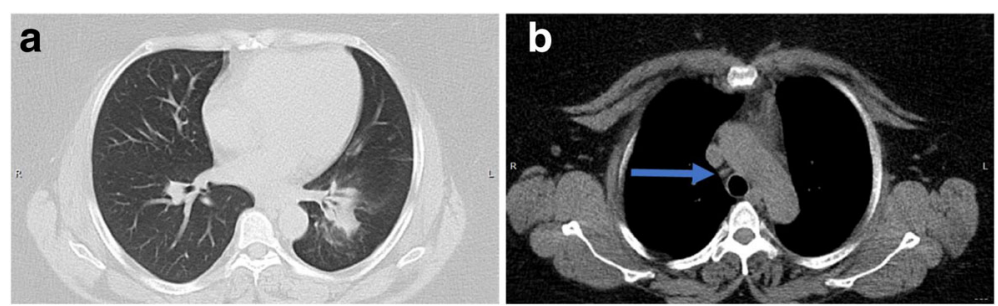

Fig. 3 Repeat chest computed tomography revealed a decrease in the size of the lung mass and lymphadenopathy. Following 3 months of high-dose prednisone therapy, repeat computed tomographic scan revealed a significant decrease in (a) the size of the lung mass, which measured $4.7 \times 3.1 \times 5.8 \mathrm{~cm}$, and (b) lymphadenopathy (arrow) 
or etanercept, have demonstrated efficacy in small observational studies $[11,12]$.

\section{Conclusions}

Atypical manifestations of pulmonary sarcoidosis are diagnostically challenging because the clinical and radiographic features of the disease mimic those of a malignancy. We aimed to illustrate a unique etiology of a lung mass, the importance of maintaining a broad differential diagnosis, and the need for a thorough evaluation. Nonetheless, with the possibility of an active malignancy, a high index of suspicion is necessary for timely diagnosis and optimal management.

\section{Acknowledgements}

Not applicable.

\section{Funding}

This work was supported by National Institutes of Health $(\mathrm{NIH})$ grant T32HL007778-21 (to CR); NIH grants HL109233, HL125850, and HL112702, and grants from the Gabriel and Alma Elias Research Fund and the Greenfield Foundation (to ELH).

\section{Availability of data and materials}

Data sharing is not applicable to this article, because no datasets were generated or analyzed.

\section{Authors' contributions}

DWK and MY reviewed the literature and prepared the manuscript. $\mathrm{RH}$ prepared Fig. 2 and the manuscript. CR and ELH managed the patient, reviewed the literature, and prepared the manuscript. All authors read and approved the final manuscript.

\section{Ethics approval and consent to participate}

Not applicable.

\section{Consent for publication}

Written informed consent was obtained from the patient for publication of this case report and any accompanying images. A copy of the written consent is available for review by the Editor-in-Chief of this journal.

\section{Competing interests}

The authors declare that they have no competing interests.

\section{Publisher's Note}

Springer Nature remains neutral with regard to jurisdictional claims in published maps and institutional affiliations.

\section{Author details}

${ }^{1}$ Department of Internal Medicine, Section of Pulmonary, Critical Care and Sleep Medicine, Yale University School of Medicine, 300 Cedar Street, TAC 441 South, P.O. Box 208057, New Haven, CT 06520, USA. ²Department of Pathology, Yale University School of Medicine, New Haven, CT, USA.

\section{Received: 15 August 2017 Accepted: 22 February 2018}

Published online: 13 April 2018

\section{References}

1. Baughman RP, Culver DA, Judson MA. A concise review of pulmonary sarcoidosis. Am J Respir Crit Care Med. 2011;183(5):573-81.

2. lannuzzi MC, Rybicki BA, Teirstein AS. Sarcoidosis. N Engl J Med. 2007:357(21):2153-65.

3. Criado $E$, et al. Pulmonary sarcoidosis: typical and atypical manifestations at high-resolution CT with pathologic correlation. Radiographics. 2010;30(6):1567-86.
4. Margaritopoulos GA, et al. Sarcoidosis in a 65-year-old woman presenting with a lung mass and pericardial effusion: a case report. J Med Case Rep. 2012;6:259.

5. Gotway $M B$, et al. Sarcoidosis presenting as an enlarging solitary pulmonary nodule. J Thorac Imaging. 2001;16(2):117-22.

6. Kachalia AG, et al. Rare coexistence of sarcoidosis and lung adenocarcinoma. Respir Med Case Rep. 2014;12:4-6.

7. Ramadas $P$, et al. Lung cancer or sarcoidosis: a diagnostic dilemma. J Cancer Ther Res. 2016:5:2

8. Ravaglia C, et al. Sarcoid-like lesion is a frequent benign cause of lymphadenopathy in neoplastic patients. Eur Respir J. 2013:41(3):754-5.

9. Tchernev $G$, et al. Sarcoidosis vs. sarcoid-like reactions: the two sides of the same coin? Wien Med Wochenschr. 2014:164(13):247-59.

10. Moller DR. Rare manifestations of sarcoidosis. ERS Monograph. 2005;32:233-50.

11. Judson MA. Advances in the diagnosis and treatment of sarcoidosis. F1000Prime Rep. 2014;6:89.

12. Brito-Zeron $P$, et al. Sarcoidosis: an update on current pharmacotherapy options and future directions. Expert Opin Pharmacother. 2016;17(18):2431-48.
Submit your next manuscript to BioMed Central and we will help you at every step:

- We accept pre-submission inquiries

- Our selector tool helps you to find the most relevant journal

- We provide round the clock customer support

- Convenient online submission

- Thorough peer review

- Inclusion in PubMed and all major indexing services

- Maximum visibility for your research

Submit your manuscript at www.biomedcentral.com/submit 